\title{
Quantification of electric field strength of tDCS in Alzheimer's and mild cognitive impairment patients
}

\author{
Utkarsh Pancholi ${ }^{1 *}$ and Vijay Dave ${ }^{2}$ \\ Assistant Professor, Department of Biomedical Engineering, L. D College of Engineering, Ahmedabad-380015, \\ Gujarat Technological University, India ${ }^{1}$ \\ Assistant Professor, Department of Biomedical Engineering, Government Engineering College, Sector-28, \\ Gandhinagar-382028, Gujarat Technological University, India ${ }^{2}$
}

Received: 18-July-2021; Revised: 24-September-2021; Accepted: 25-September-2021

(C)2021 Utkarsh Pancholi and Vijay Dave. This is an open access article distributed under the Creative Commons Attribution (CC BY) License, which permits unrestricted use, distribution, and reproduction in any medium, provided the original work is properly cited.

\begin{abstract}
The aging brain causes the problems associated with decision making, memory loss, language problems, personality problems, and changes in behavior. Physicians decide treatment based on disease progression and the patient's overall health. Alzheimer's disease (AD) and Mild Cognitive Impairment (MCI) are two majorly reported clinical abnormalities in today's time. The adversity of the disease can be controlled with timely diagnosis and choice of treatment modality. Noninvasive treatment like transcranial electrical stimulation has shown effective results in drug-resistant and early diagnosed patients. Transcranial Direct Current Stimulation (tDCS) uses low electrical direct current through specialized stimulating electrodes. The induced electric field within the targeted area can be mea sured in vivo with greater accuracy with its limitation of applicability to humans. Characteristics of the tissue changes with its compositional variation with different tissue mass like Cerebrospinal Fluid (CSF), Skull, Skin, Gray matter, and White matter. Computational modeling of tissue characteristics and external stimulation provides a better solution for the effective measurement of spatial electric field distribution. The effectiveness of treatment greatly depends on targeted electrical stimulation with precise localization of stimulation electrodes. Apart from the location of the stimulation electrodes, electrode size, shape, duration of stimulation, the patient's specific anatomy, the strength of the current, and conductivity of tissue alter the treatment efficacy and clinical outcome. In this study, we have obtained Magnetic Resonance Imaging (MRI) images from the AD neuroimaging initiative to create patient-specific head models for AD and MCI patients. Simulation of Non-invasive Brain Stimulation (SimNIBS) open-source software used for calculating electric field induced by transcranial electrical stimulation. Obtained results were compared for both patient groups to know the variation of electric field distribution across the head regions. Results suggest that electric field distribution varies with selected stimulation parameters and patient-specific head models. Increasing current intensity by $25 \%$ of $1 \mathrm{~mA}$ results in a $25 \%$ increase in electric field strength, whereas a $50 \%$ increase in $1 \mathrm{~mA}$ of current intensity results in a nearly $49.46 \%$ increase in electric field strength of Left Dorsolateral Prefrontal Cortex (LDLPFC). With standard conductivities of head tissues and uniform stimulation parameters for both the patients, we obtained varied electric field strength which signifies the tissue abnormality caused by neurodegenerative disease.
\end{abstract}

\section{Keywords}

Transcranial direct current stimulation (tDCS), Alzheimer's disease, Mild cognitive impairment, Electric field distribution.

\section{Introduction}

Alzheimer's and dementia is rapidly increasing health issue in aging people. It is expected that 900 crores of people will cross 60 years of their age by 2050 across the world. Aging is one of the factors for Neurological diseases mostly in a developing country like India because of the lack of ease of living.

\footnotetext{
*Author for correspondence
}

1168
As per the statistics for age, 7.5 crores of people will be suffering from Alzheimer's and dementia-related neurological problems by 2030 [1]. India is having only 0.010 mental health hospitals per 1 lakh population. Mild Cognitive Impairment (MCI) is another neurodegenerative disease considered as an intermediate stage between the early normal cognitive ability to clinical dementia. Patients with clinical evidence of MCI are expected to develop dementia and Alzheimer's disease in a short period [2]. Early symptoms of MCI include forgetting things, inability 
to find words for communication, personality changes are likely to develop Alzheimer's disease where these symptoms get worsen. Alzheimer's patients have more problematic symptoms associated with language problems, complete memory loss, and severe personality behavior. To eliminate the social stigma of mental illness, it is an urgent need to prevent and treat these diseases with appropriate treatment modalities.

Transcranial electrical brain stimulation finds more attention in the neuroscience and Neuro-rehabilitation community because of its non-invasive behavior. Other non-invasive treatment modalities for neurodegenerative diseases are Electro-Convulsive Therapy (ECT), Transcranial magnetic stimulation, and Transcranial direct current stimulation. All these techniques utilize delivery of current trans-cranially where no surgical interventions are required. Direct shock therapy like ECT delivers 70 to 120 volts to the patient's head increases mortality risk between 2 to 10 patients per 100000 therapies [3]. ECT also has adverse effects on cardiac and respiratory functions. Vagus nerve stimulation, an invasive treatment modality for epilepsy and depression is also having side effects of vocal cord paresis, arrhythmia, and facial weaknesses [4]. In recent times transcranial direct current stimulations have been receiving much interest among Neuroscientist and neuropsychiatrists for their less adversity to patients.

Transcranial direct current stimulation is most suitable for drug-resistant neurological patients. Transcranial Direct Current Stimulation (tDCS) alters brain activity by modulating depolarization and hyperpolarization within the specific regions of target therapy. Using two stimulating electrodes tDCS can depolarize and hyperpolarize the target area of the brain by anodal and cathodal stimulation respectively. Concerning the Transcranial Magnetic Stimulation (TMS), tDCS does not induce action potentials rather it augments neuronal excitability using Long Term Potentiation (LTP) upon anodal stimulation and Long Term Depression (LTD) upon cathodal stimulation [5]. TMS is considered as supra-threshold stimulation while tDCS is considered as sub-threshold stimulation technique. Delivered current can alter the concentration of calcium $(\mathrm{Ca}+)$ ions into targeted brain regions [6]. Neurotransmitters, Gamma-Aminobutyric Acid (GABA), and glutamate can be increased and decreased upon the anodal and cathodal tDCS stimulation respectively [7]. Marceglia et al. [8] found increased memory associated with better recognition of words reflected in Electroencephalography (EEG) waves in the Alzheimer patient group by tDCS stimulation. With various behavior and cognitive assessment techniques, Meinzer et al. [9] concluded improvement in memory in the MCI patient group after tDCS stimulation. By delivering current between 1 and $2 \mathrm{~mA}$ at targeted brain regions will induce electric field distribution within and near the stimulation area. Characteristics of induced electric field distribution depend on brain tissue's anatomical and physiological condition. Also, electric field distribution in the targeted brain regions is heavily altered by individual anatomy, the strength of the direct current, electrode's size and shape, tissue conductivity parameters, and electrode placements [10]. Variation in brain anatomy person to the person demands an individual approach of stimulation protocol to achieve treatment goals.

Bran stimulation requires accurate information regarding location and stimulation parameters to obtain optimum therapeutic effects. Alteration of electric field strength due to subjective variation can be estimated with computational approaches. The subjective approach to finding out the right therapy model before giving the actual treatment with the tDCS device motivates this study. To address the challenges like 1 . How to simulate a customized treatment model? 2. What parameters have to be considered for simulation study? 3. How to interpret electric field distribution in brain tissues 4 . What is the effect of changing the stimulation parameters? Conventionally current reaching to the brain tissue, measured by deep sited microelectrode which is impractical to study changes due to stimulation variation. Computational platforms like the realistic, volumetric approach to Simulate Transcranial electric stimulation (ROAST) and SimNIBS offer an estimation of electric field strength due to the current flowing within the targeted brain tissue. Rodella et al. [11] opinioned about the inter-subject uniqueness of tissue characteristics requires modification in stimulation parameters to check therapy progress. The objective of this study is to check electric field strength quantification for the same stimulation protocol for two diseased subjects. With the simulation platform, the dose required for therapy can be decided with optimum stimulation parameter selection. Simulation of tDCS in computational platform gives a clear idea about the path of the current flowing through the brain tissue and resulting electric field strength generated. 


\section{Literature review}

Electric field distribution patterns upon tDCS active stimulation rely on anatomical and physiological individuality.

Indahlastari et al. [12] conducted a study on 587 older age healthy people for estimating the amount of applied transcranial direct current reaching the neural tissues. They modeled tDCS with two electrodes (F4 and $\mathrm{C} 3$ as an anode, F3 and FP2 as a cathode) with other stimulation parameters (Current intensity: $2 \mathrm{~mA}$, Electrode size: $5 \times 7 \mathrm{~cm}^{2}$, standard conductivities using ROAST and reported the current carrying capacity of the neural tissue reduces due to different brain ratios (Measure of Brain Atrophy). Other tissues of the head like Skull, CSF, White Matter, Gray Matter, and Skin restricts current reaching to the target location of stimulation as they contain some resistivity of their own. Structural MRI predicts anatomical variability within a diseased brain. In line with anatomical changes across the brain tis sue, there is a possibility of functional changes in other physiological systems.

Vasavada et al. [13] studied disability associated with the function of smell (Olfactory dysfunction) with functional magnetic resonance imaging (fMRI) and compared results among three subject groups (Normal cognitive, AD, MCI). The comparis on showed normal cognitive subjects had significantly more activated brain tissue in the primary cortex than $\mathrm{AD}$ and $\mathrm{MCI}$ subject groups.

Wang et al. [14] discovered a novel approach to know the effects of the multi-treatment model (Multiple combinations of tDCS and Repetitive Transcranial Magnetic Stimulation (rTMS) on five healthy subjects with stimulation parameters (Electrode-position: C4cathode, Fp1-anode, current intensity: $1 \mathrm{~mA}$, size: $5 \times$ $7 \mathrm{~cm}^{2}$, standard tissue conductivities) and reported increase in cortical excitability, modeled using SimNIBS. Relative positions of a coil (rTMS) and electrodes (tDCS) showed the change in electric field distribution in a simulated model in SimNIBS which can modulate cortical excitability in healthy brains. Drug-resistant patients with Major Depressive Disorder (MDD) show reduced symptoms with tDCS treatment [15].

Hill et al. [16] applied tDCS stimulation on 20 healthy individuals to know the therapeutic outcomes for working memory analysis with stimulation parameters (Electrode-position: F3- anode, Fp1-F7-C3, and Fzcathode, current intensity: $1.5 \mathrm{~mA}$, size: $3.14 \mathrm{~cm}^{2}, 15$ $\mathrm{min}$ ) and reported findings that working memory of the healthy individual can be altered with tDCS stimulation which resulted from increased neural plasticity. 10-20 Electrode placement method for precise tDCS stimulation eases target localization. Computational approaches to estimate Electric Field Distribution and its variability signifies Electric Field Variation in cortical regions. One-size-fits all approach of conventional tDCS machine delivers currents in the range of 1 to $2 \mathrm{~mA}$. Estimation of delivered currents in target locations varies upon individual tis sue conductivities of neural tis sues.

Laakso et al. [17] experimented with tDCS on 28 healthy individuals with no previous history of epileptic seizures with stimulation parameters (Electrode-position: contralateral orbit -cathode, Hand M1-anode, current intensity: $1 \mathrm{~mA}$, size: $5 \times 5 \mathrm{~cm}^{2}, 20$ $\mathrm{min}$ ) and concluded their experiment on how prior EF modelling can reduce patient-specific variation. Cortical excitability depends on the type of stimulation given to the patient's head i.e. anodal (Increased excitability) or cathodal (decreased excitability). The computational approach gives an estimation of an electric field generated with specific stimulation parameters for modulating the brain cortex. Physiological effects on diseased brain tissue under these stimulations require more in-vivo studies for validation discussed three different approaches for studying effects of tDCS (in-vivo, in-vitro, and insilico) where in-vivo study measures electric field on tis sues with path-clamp techniques, in-vitro studies on mice indicated long term effects on hippocampal plasticity [18]. Also, effects of voltage variation on transmembrane potential have been studied by various neuron models like Hodgkin-Huxley model [19], integrate and fire model [20], and other compartmental models for neurons. However, resting membrane potential in neurons is in the order of $0.2 \mathrm{mV}$ which can be easily achieved by sub-threshold tDCS stimulation in the range of 1-2 mA current intensity [18].

Lee et al.[21] studied three realistic head models generated from SimNIBS using MRI images for maximum current optimization and reported deeper structures of the brain can be stimulated with computational modelling without affecting the neocortical neurons.

As individualized or patient-specific Non-invasive Brain Stimulation (NIBS) results in optimum therapy effects, it is required to test computational simulation to avoid unnecessary direct current stimulation on healthy or undesired tissues during actual tDCS 
operation. Parameters for stimulation can be changed and checked for effects with graphical representation. Shunted currents can be visualized with SimNIBS by separating associated brain tissues. Visualization of electric field distribution with varying stimulation parameters like electrode size, shape, stimulating direct current intensity, tissue conductivities can be utilized to plan the treatment. The location of the electrode can be decided by first identifying a region of the brain and knowing a corresponding point on the 10-20 electrode placement method generally used for EEG. In this study, we have identified Left Dorsolateral Prefrontal Cortex (LDLPFC) location for the stimulation for $\mathrm{AD}$ and MCI patients with various literature surveys. Two patients have been identified and structural MRI images were taken from the Alzheimer's Disease Neuroimaging Initiative (ADNI) platform. With desired stimulation parameters simulation has been done with SimNIBS.

\section{Materials and methods}

\subsection{Methodology approach}

To generate patient-specific tetrahedral head models (finite element model), T1 weighted MRI images were used from an open-source platform. With short repetition time (TR) and time to echo (TE), contrast and brightness can be varied with $\mathrm{T} 1$ properties of tissue. In $\mathrm{T} 1$ weighted images, tissues appear differently in contrast (CSF- Dark, White matter-
Light, Cortex- Gray, Fat-Bright, Inflammation, Infection, and Demyelination-Dark). To differentiate various tis sue properties like conductivity and density image segmentation has to be done on MRI images. Pre-processing, segmentation, and post-processing of MRI images were done with the SimNIBS software in an automated manner. Creating head mesh from T1 weighted image from NifTI (Neuroimaging Informatics Technology Initiative) files results in a tetrahedral matrix which is a three-dimensional (3D) finite element model of MRI image. tDCS stimulation requires parameter selection for size, shape, and location of the electrode, the conductivity of the tis sue, and current intensity. An induced electric field can be visualized in an Open-source 3D finite element mesh generator (gmsh) platform inbuilt with SimNIBS pipeline. We have considered current intensity as a prime controlling parameter to analyze electric field distribution in two diseases by keeping other parameters like location, size, and shape of the electrode and thickness of the electrodes constant. Analysis of resulted electric field distribution among various brain tissue has been done with this study. Further electric field distribution among different tissues (CSF, skin, skull, white-matter, and graymatter) within the brain can be separated with special functions available in SimNIBS. Figure 1 shows a block diagram of the process followed.

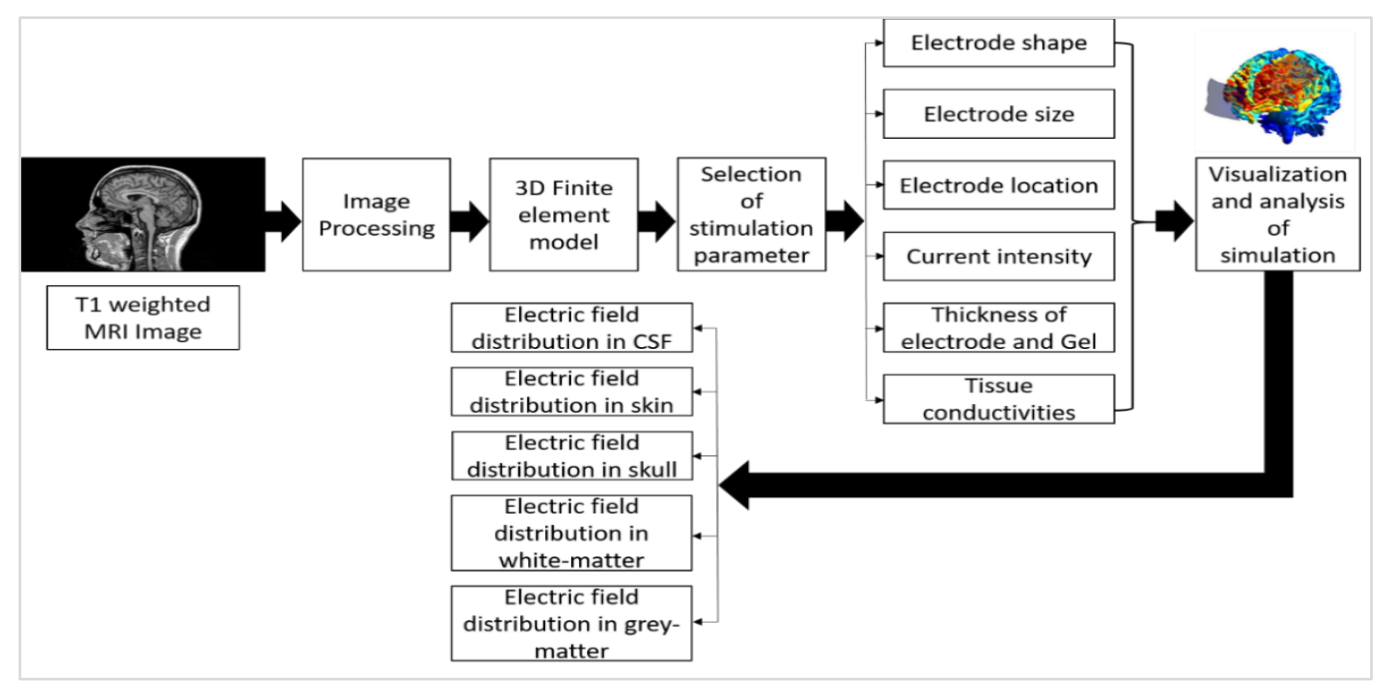

Figure 1 Block-diagram for simulation process

3.2Magnetic resonance imaging (MRI) image preparation

Structural MRI images were obtained from ADNI (https://ida.loni.usc.edu/login.jsp) for two diseased groups (One for Alzheimer's disease Patient, Male,
Age- 64 and One for Mild Cognitive Impairment Patient Female, Age-63). Imaging protocols for Alzheimer's patients (Subject-1) \& for Mild cognitive impairment patients (Subject-2) are as shown in Table 
1. A complete list of abbreviations is shown in Appendix I.

Table 1 Imaging protocols for MRI

\begin{tabular}{ll}
\hline Imaging protocols & $\begin{array}{l}\text { AD } \\
\text { Patient }\end{array}$ \\
\hline Acquisition Plane & SAGITTAL \\
\hline Acquisition Type & Coil=SENSE-head \\
\hline Field Strength & 3.0 (tesla) \\
\hline Flip Angle & 8.0 (Degree) \\
\hline Manufacturer & Philips Medical Sy stems \\
\hline Mfg. Model & Intera Achieva \\
\hline Matrix X & 256.0 (pixels) \\
\hline Matrix Y & 256.0 (pixels) \\
\hline Matrix Z & 170.0 (pixels) \\
\hline Pixel Spacing & X=1.0 Millimetre (mm) \\
\cline { 2 - 2 } & Y=1.0 mm \\
\hline Pulse Sequence & GR \\
\hline Slice Thickness & $1.2 \mathrm{~mm}$ \\
\hline TE & $3.2 \mathrm{Millisecond} \mathrm{(ms)}$ \\
\hline TI & $0.0 \mathrm{~ms}$ \\
\hline TR & $6.8 \mathrm{~ms}$ \\
\hline Weighting & T1 \\
\hline
\end{tabular}

3.3Image processing and creating head mesh To create individualized head mesh or volume conductor mesh for simulating induced electric field strength, SimNIBS offers two different process pipelines [22]. Which uses Statistical Parametric Mapping (SPM) and Computational Anatomy Toolbox (CAT) for segmenting different tis sues in the brain and neck regions into specific voxels. First, brain regions like CSF, White Matter (WM), Gray Matter (GM), Ventricles, Cerebellum, Skull, and Skin have to be segmented followed by surface generation for separating the different regions. The separated hemisphere can be re-joined by adding corpus callosum. And finally creating tetrahedral volume mesh by removing overlapping and intersections [23]. Mesh quality can be increased by increasing the number of nodes per unit area. SimNIBS offers a headreco pipeline for creating head mesh which comprises 0.5 nodes per $\mathrm{mm} 2$. Which can be modified with defined programming. Generated head mesh can be viewed in gmsh for defining stimulation parameters and identifying the targeted area.

\subsection{Parameter selection for tDCS stimulation}

Non-drug electric therapy demands more attention on stimulation protocols to be followed. Current intensity, shape-size (Rectangular-elliptical, 5×5-5×7 $\mathrm{cm}^{2}$ ) of electrodes, precise location, electrode composition with conductive gel or sponge determines the effectiveness of treatment. Direct current can be administered non-invasively through specialized electrodes using the 10-20 electrode placement method [24]. Damage to certain parts of the brain results in mild to severe changes in various human activities. The frontal lobe is associated with speech, language, personality, social behavior, decisionmaking [25]. The parietal lobe is responsible for the spatial distribution, relative position of objects, differences in shape and size, and also learning [26]. The temporal lobe decides the ability to remember things (All kinds of memory), language forming, and speech perception [27]. The occipital lobe is concerned about vision and vision-related problems like color blindness [28]. Figure 2 shows various lobes of the brain. Patients with AD and MCI disease are having difficulties with cognitive skills like changes in social behavior, inability to express language, attention deficiency, and altered thinking. Region identification for stimulation varies treatment efficacy and generated electric field locally [29-32]. Location for NIBS is determined by corresponding or closest montage of 10-20 electrode placement method. LDLPFC shows promising results upon tDCS stimulation for patients with Alzheimer's and Mild cognitive impairment [33-35]. A further position of the anodal and cathodal electrode was decided by identifying the location concerning the 10-20 electrode placement method. Electrodes F3 (Frontal) (Anodal) and Fp2 (Frontal-parietal) near to supraorbital area (Cathodal) identified according to literature review for Alzheimer's Patient and MCI patients [36]. Rectangular stimulating electrodes of the size $5 \times 7 \mathrm{~cm}$ (Figure 3) with the direct current intensity of $1.75 \mathrm{~mA}$ are used as a simulation parameter. Blue colored surface in Figure 3 shows the electrode surface of the thickness $1 \mathrm{~mm}$, the base of the electrode (white color) indicates the thickness of the gel $(5 \mathrm{~mm})$. Size and shape of electrode and location of cathode greatly influence patterns of electric field distribution for both focality and strength. If electrode size chosen for stimulation decreases, resulting in precise and focal current distribution [37]. Moreover, the direction of current flow depends on the position of the cathode, change in the location of the cathode ultimately changing brain tissues under current stimulation [38]. Electrode gel layer considered as $5 \mathrm{~mm}$ to generate actual resistance offered by Electrode-electrolyte medium. Figure 4 shows the placement of a customized electrode on the skull (Figure 4 (A) and (C)) and the gray matter (Figure 4 (B) and (D)) for better understating of the stimulation target. Before the simulation placement of electrodes on skull and gray matter can be visualized with Figure 4. Current intensity at the cathode must be opposite to anodal current intensity as $-1.75 \mathrm{~mA}$. 
Modeling of the electrode is done by SimNIBS platform by iteration method where surfaces of skin and electrode formed for realistic simulation model [39]. Table 2 and Table 3 shows Electrode parameter selection for $\mathrm{AD}$ and $\mathrm{MCI}$ patient respectively.

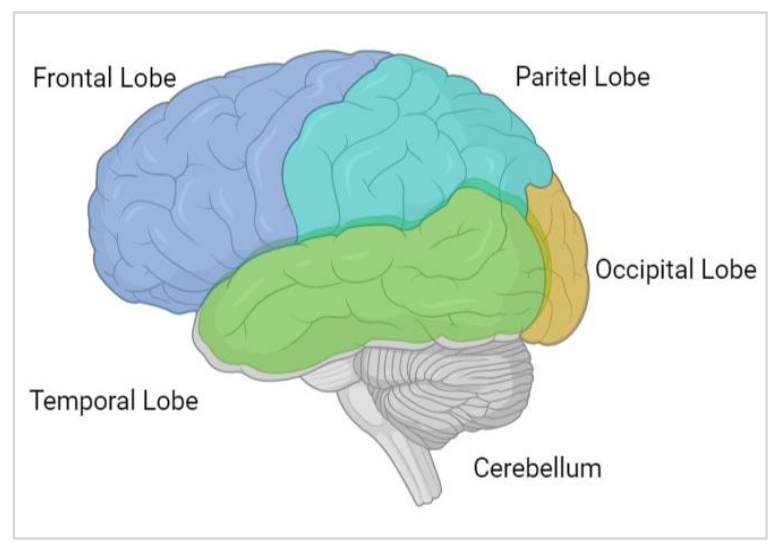

Figure 2 Various lobes of the brain (Created with BioRender.com)

3.5Conductivity values and quantification of generated electric field

Electric field distribution in a human body varies on bioelectric sources and conductivity values of the tissues [40]. As brain and associated structures do not have homogeneity in tissue conductivity it would be difficult to estimate the actual electric field distribution. For simplicity, all tissues are considered homogenous and is otropic, the conductivity of various structures of the brain are listed in Table 4. An electric field can be calculated with the equation $\vec{E}=-\nabla \varphi$ where $\vec{E}$ is an electric field vector and $\varphi$ is an electric potential. The electric potential $\varphi$ was computed using an electrostatic formulation with Dirichlet boundary conditions at the electrode connectors set to fixed potential values [41]. Current density can be calculated via Ohm's Law, $\bar{J}=\sigma \vec{E}$, where $\sigma$ is an electrical conductivity and $\vec{E}$ is an electric field. Generated electric field can be characterized by two quantities i.e. magnitude (Strength of electric field) and direction of field in a given space. SimNIBS is having limitation of quantification of electric field as it can only show magnitude of the electric field. As quantum or strength of the electric field is always positive, we get some integer value in the form of electric field or norm.

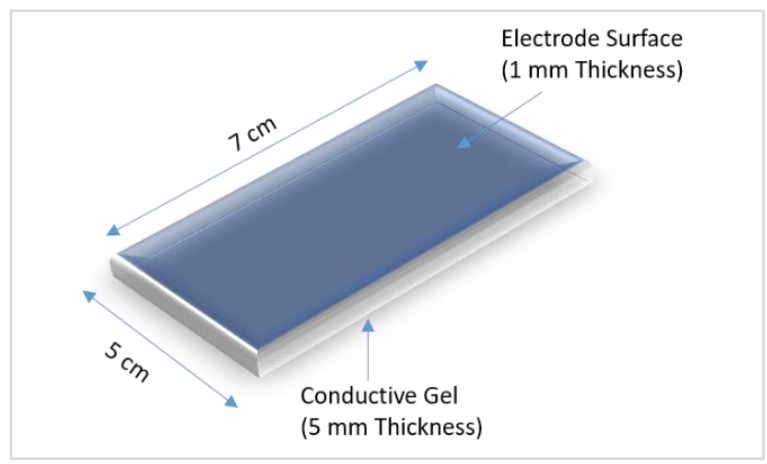

Figure $35 \times 7 \mathrm{~cm}^{2}$ electrode of $1 \mathrm{~mm}$ thickness with 5 $\mathrm{mm}$ gel thickness

Table 2 Stimulation parameter selection for an AD patient

\begin{tabular}{|c|c|c|c|c|c|c|c|c|c|c|}
\hline \multicolumn{11}{|c|}{ Stimulation parameter selection (AD patient) (Patient-specific) } \\
\hline \multirow[b]{2}{*}{$\begin{array}{l}\text { Electrode } \\
(10-20 \\
\text { Electrode } \\
\text { placement } \\
\text { method }\end{array}$} & \multicolumn{6}{|c|}{ Coordinates } & \multirow[t]{2}{*}{ Shape } & \multirow[t]{2}{*}{ Size } & \multirow{2}{*}{$\begin{array}{l}\text { Intensity of } \\
\text { stimulation }\end{array}$} & \multirow{2}{*}{$\begin{array}{l}\text { Thickness } \\
\text { of electrode }\end{array}$} \\
\hline & Refere & ice coord & nates & Actual & oordina & & & & & \\
\hline F3 & -42.20 & 65.09 & 50.38 & -42.20 & 55.09 & 50.38 & Rectangular & $\begin{array}{l}5 \times 7 \\
\mathrm{~cm}^{2}\end{array}$ & $1.75 \mathrm{~mA}$ & $\begin{array}{l}\text { Electrode } \\
\text { Thickness:1 } \\
\text { mm, } \\
\text { Gel } \\
\text { Thickness: } 5 \\
\text { mm }\end{array}$ \\
\hline Fp2 & 38.99 & 95.52 & 15.06 & 28.99 & 95.52 & 15.06 & Rectangular & $\begin{array}{l}5 \times 7 \\
\mathrm{~cm}^{2}\end{array}$ & $-1.75 \mathrm{~mA}$ & $\begin{array}{l}\text { Electrode } \\
\text { Thickness: } \\
1 \mathrm{~mm}, \\
\text { Gel } \\
\text { Thickness: } 5 \\
\text { mm }\end{array}$ \\
\hline
\end{tabular}




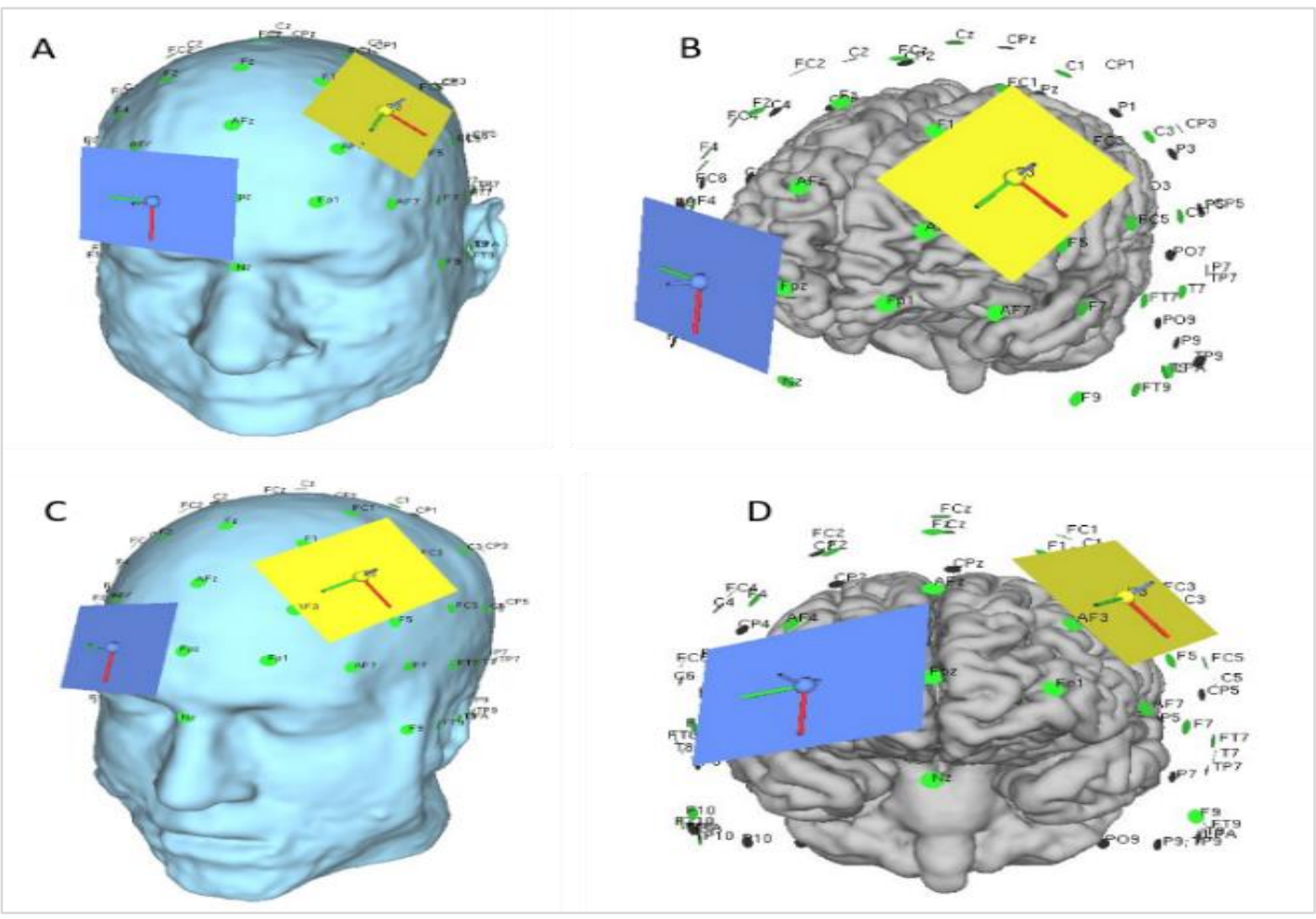

Figure 4 A) Placement of electrode on AD patient, B) Shows gray matter of AD patients head model with a placed electrode, C) Placement of electrode on MCI patient, D) Shows gray matter of MCI patients head model with placed electrode

Table 3 Stimulation parameter selection for MCI patient

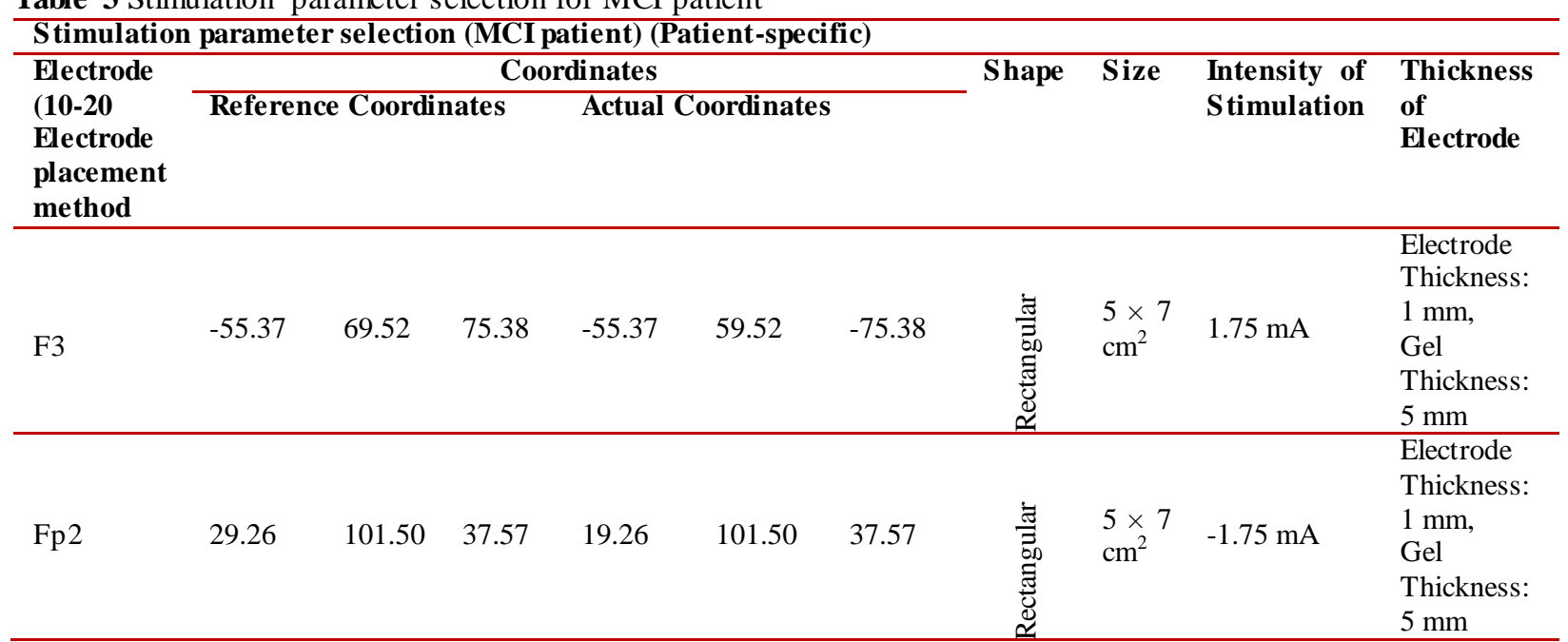


Table 4 Standard conductivity values

\begin{tabular}{ll}
\hline Tissue name & $\begin{array}{l}\text { Conductivity value } \\
(\mathbf{S} / \mathbf{m})\end{array}$ \\
\hline White Matter & $0.126[42]$ \\
\hline Gray Matter & $0.275[42]$ \\
\hline Cerebrospinal fluid & $1.654[42]$ \\
\hline Bone & $0.01[42]$ \\
\hline Scalp & $0.465[42]$ \\
\hline Eyes & $0.5[41]$ \\
\hline Silicon Rubber & $29.4[43]$ \\
\hline Gel & $1.5[43]$ \\
\hline
\end{tabular}

\section{Results}

Firstly, the head model was created with tetrahedral mesh for further processing of simulation. Segmented images can be shown in SPM. Boundaries across the structures can be shown with clear segmentation. In Figure 5 section-A shows a T1-weighted scan coregistered with the Montreal Neurological Institute (MNI) template which can differentiate between soft tissues like white and gray matter and skull as well, Section-B shows the result in SPM after deselecting the T1-weighted scan. In this way differentiation among the brain, structures can be identified properly as well the effectiveness of the segmentation process can be characterized. In the next step, we investigate electric field strength in both AD and MCI patients. Considering stimulation parameters selected in (Table 2 and Table 3) for AD and MCI patients respectively we obtained electric field distribution across the head model. While inspecting the simulation we found that electric field strength in MCI patients (0-0.348 Volts per meter $(\mathrm{V} / \mathrm{m})$, Figure $6(\mathrm{~A}))$ is slightly higher than in the AD patient $(0-0.328 \mathrm{~V} / \mathrm{m}$, Figure $6(\mathrm{~B}))$.

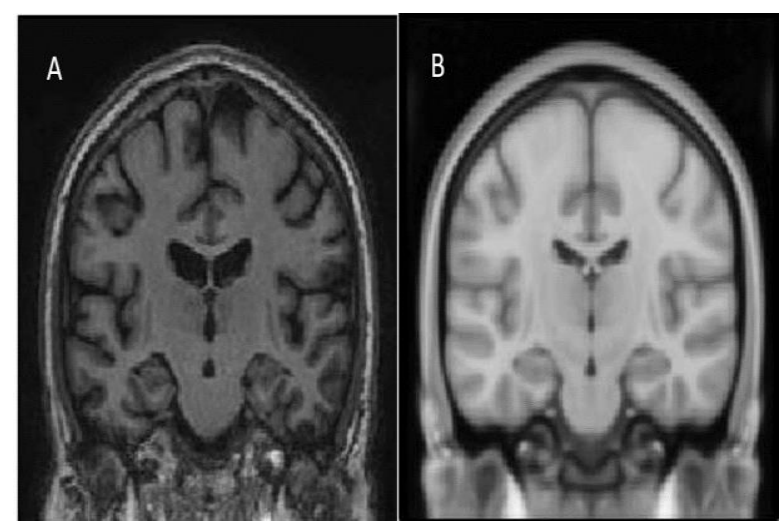

Figure 5 Displayed Data upon check option A) T1weighted scan co-registered with the MNI template. B) MNI template showing result upon de-selecting the T1-weighted scan in SPM

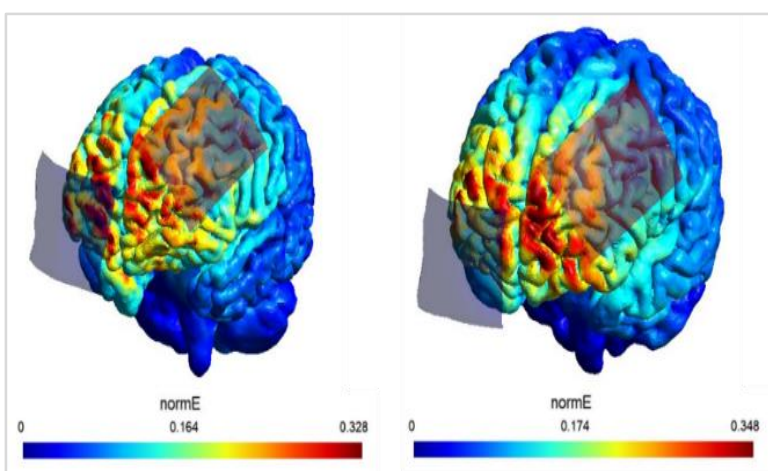

Figure 6 A) Electric field strength in AD Patient B) Electric field strength in MCI patient

Simulation results of obtained electric field distribution can be characterized based on regions. Associated structures of the brain can be visualized with SimNIBS. White matter, gray matter, cerebrospinal fluid, skull, and skin can be clipped and separated from the head mesh for a better understanding of field distribution. The majority of current shunted within the skull and other brain tis sues apart from gray matter. The dose can be adjusted to nullify the current shunting within these areas. Figure 7 showing regions of the brain with electric field distribution WM (A), CSF (B), skull (C), and Skin (D) in AD patients. Where in Figure 8 showing regions of the brain with electric field distribution WM (A), CSF (B), skull (C), and Skin (D) in MCI patients. In this way, one can find out in which region of the head there is the penetration of the electric charge. The strength of the electric field obtained can be changed by changing stimulation parameters. The orientation of electrodes can be changed with stimulation coordinates. The first line of Table 5 shows that the displayed data is the "normE", Electric field strength of the targeted region 2 referring to gray matter. Value $0.337 \mathrm{~V} / \mathrm{m}$ corresponds to the 99.9 th percentile of the total norm electric field, $0.254 \mathrm{~V} / \mathrm{m}$ to the 99th percentile, and $0.184 \mathrm{~V} / \mathrm{m}$ to the 95 th percentile. Same as in Table 6 first-line show that the displayed data is the "normE", Electric field strength of the targeted region 2 referring to gray matter. Value $0.341 \mathrm{~V} / \mathrm{m}$ corresponds to the 99.9 th percentile of the total norm electric field, $0.258 \mathrm{~V} / \mathrm{m}$ to the 99th percentile, and $0.19 \mathrm{~V} / \mathrm{m}$ to the 95th percentile. The Focality of an electric field can be measured in terms of gray matter volume with an electric field. Change in size of the electrode changes generated electric field. A total volume of the brain under the stimulation and its as sociated electric field strength can be generated with the volume vs. EF graph. Minimum EF is indicated as blue color and maximum EF is indicated as red color. 
The total electric field ranges between $0-0.45 \mathrm{~V} / \mathrm{m}$. Figure 9 (A) and Figure 10 (A) show Electric field strength distribution in $\mathrm{AD}$ and $\mathrm{MCI}$ patients respectively. Whereas Figure 9 (B) and Figure 10 (B) show Volume Vs Electric Field (EF) graph for simulation result.
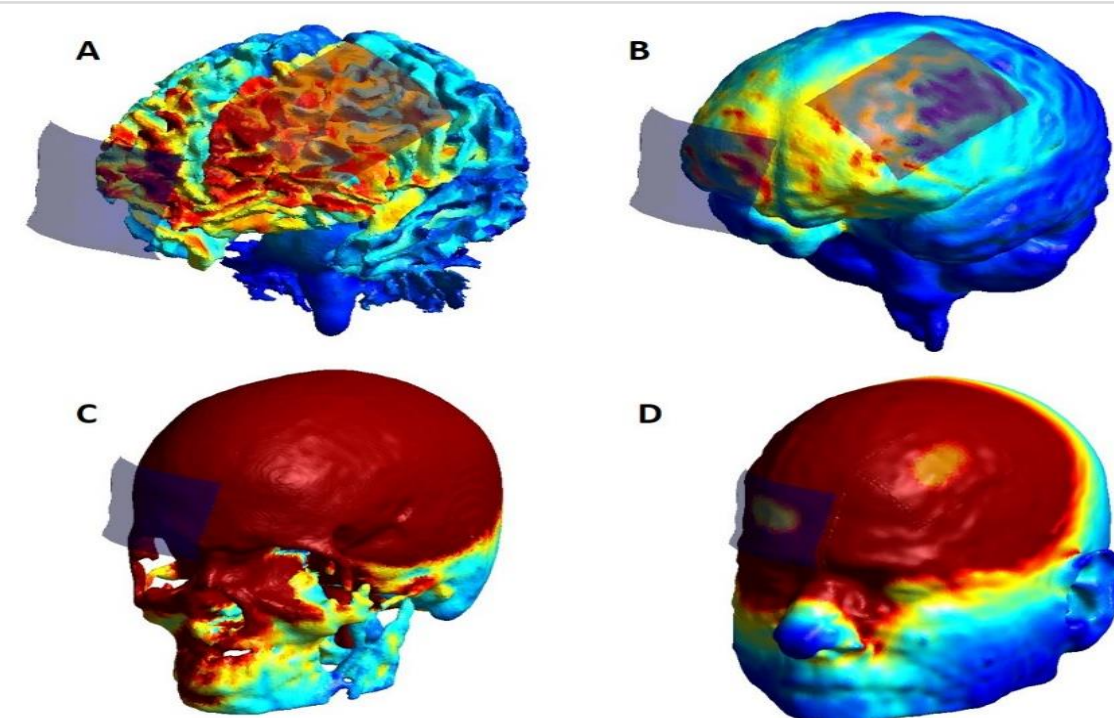

D

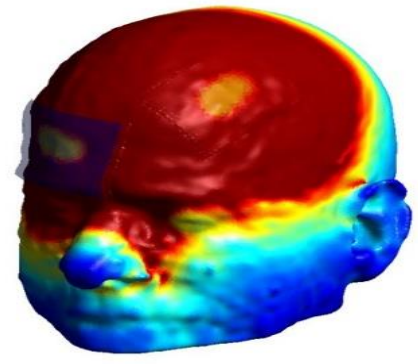

$$
0
$$

normE

0.164

0.328

Figure 7 EF distribution in WM (A), CSF (B), skull (C), and Skin (D) in AD Patient

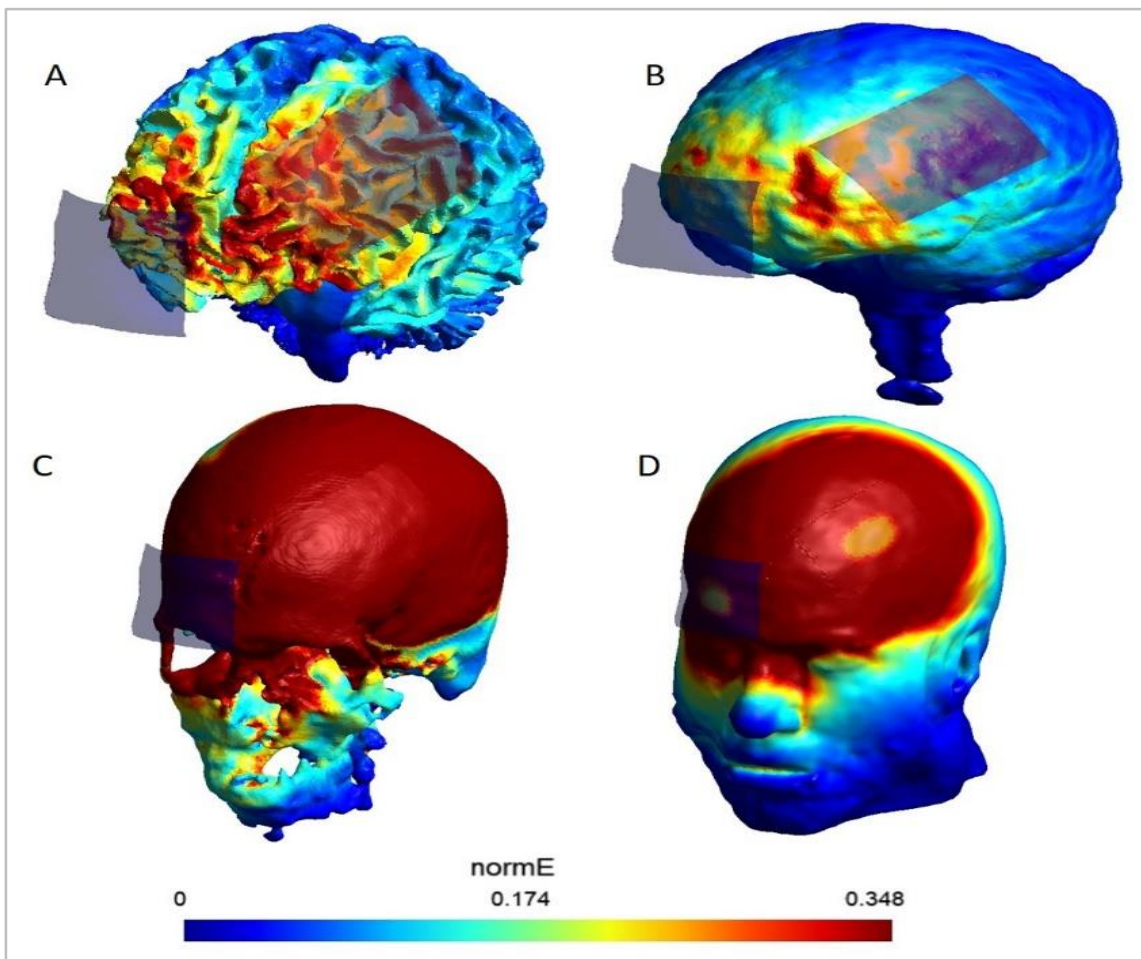

Figure 8 EF distribution in WM (A), CSF (B), skull (C), and Skin (D) in MCI Patient 


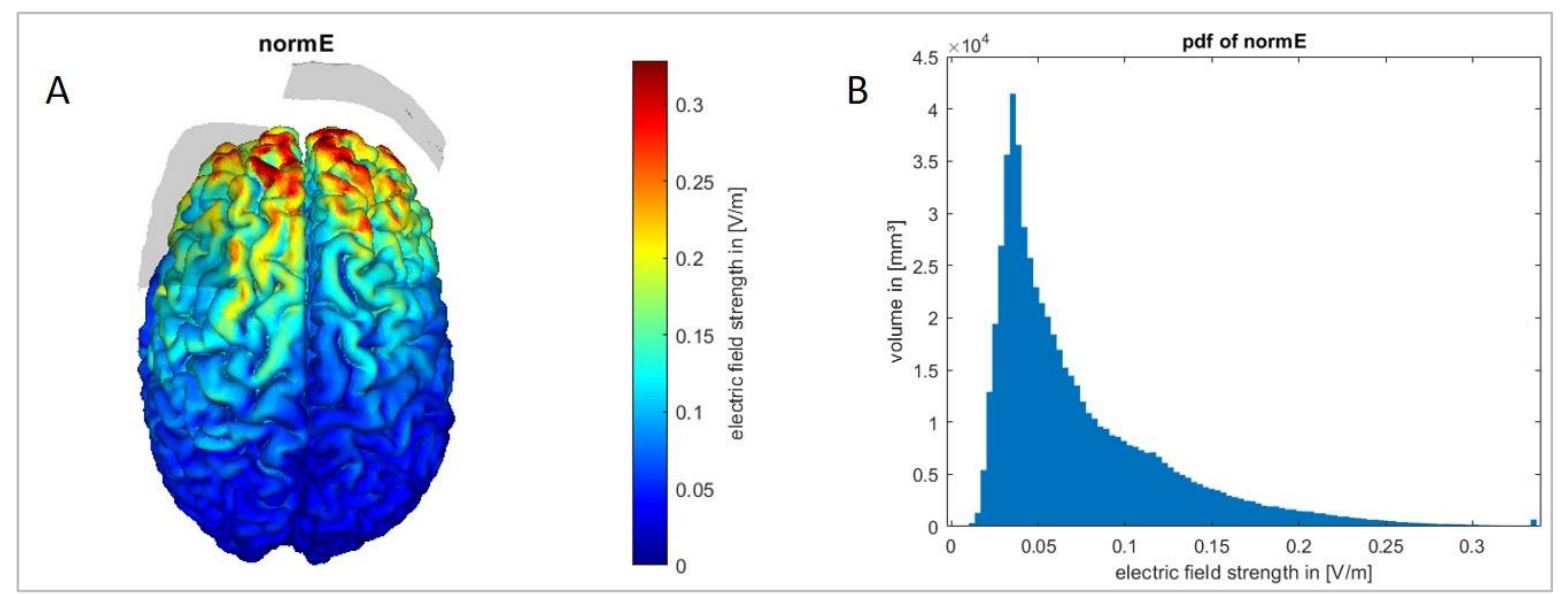

Figure 9 A) Electric field strength distribution in AD Patient B) Volume vs EF graph for simulation result

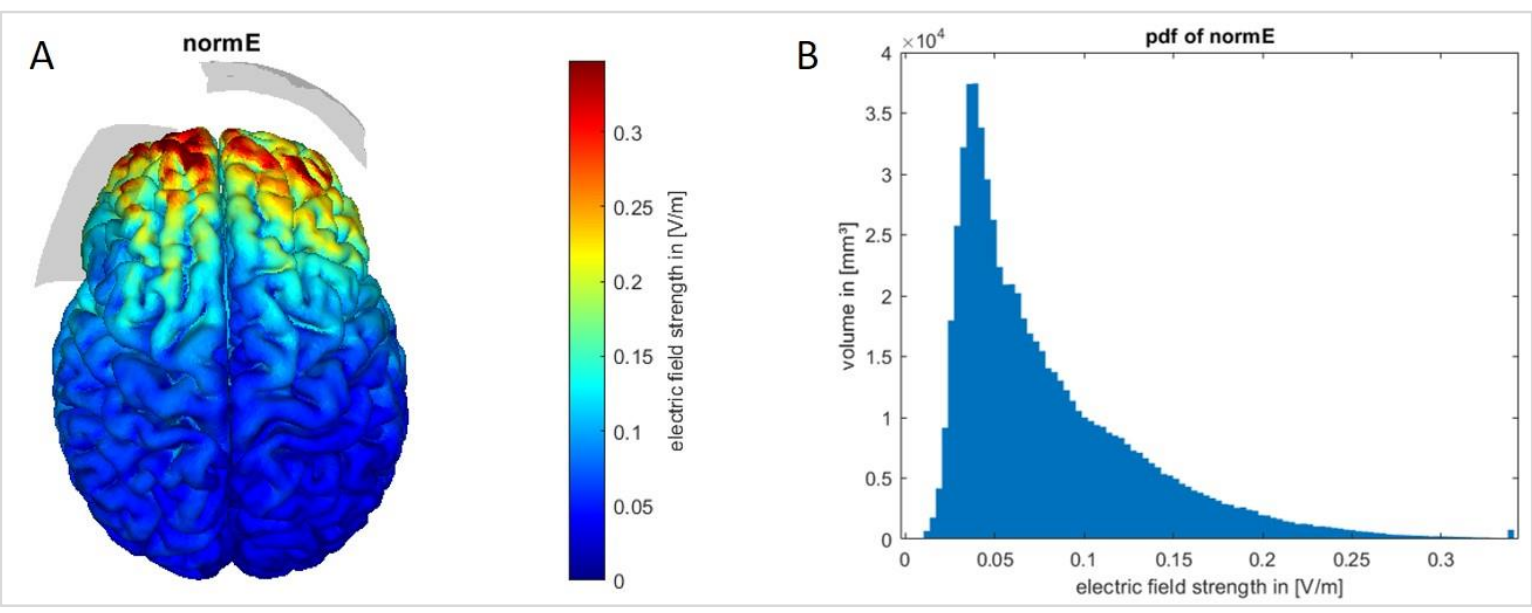

Figure 10 A) Electric field strength distribution in MCI Patient B) Volume vs EF graph for simulation result

Table 5 Output summary of tDCS stimulation in AD Patient

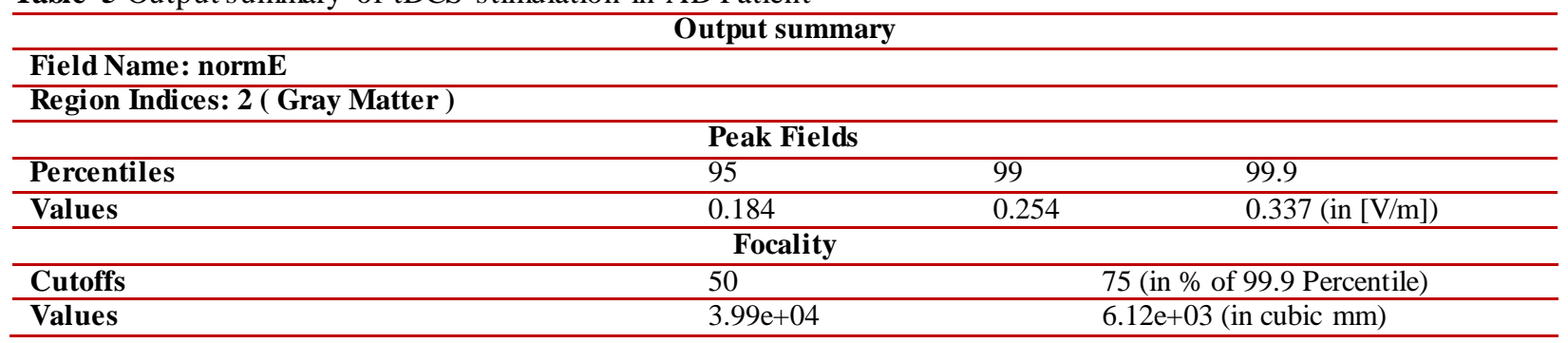

Table 6 Output summary of tDCS stimulation in MCI Patient

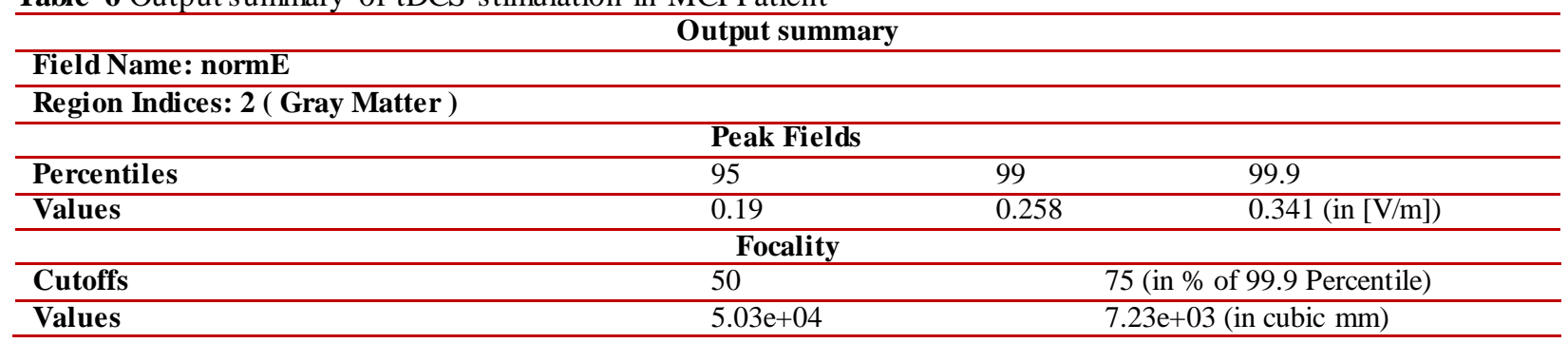


Current reaching the brain tissue varies upon dose resulting in altered cortical excitability. Changing the intensity of delivered current alters Electric Field Distribution at a target location. Current intensities selected for dose are between 1 to $2 \mathrm{~mA}$. We have considered $\mathrm{AD}$ patient to check $\mathrm{EF}$ variability upon increasing current intensity. $1 \mathrm{~mA}$ of direct current causes $0.188 \mathrm{~V} / \mathrm{m}$ (Figure 11 (A)) of electric field strength. Increasing current intensity to $1.25 \mathrm{~mA}$, we get $0.235 \mathrm{~V} / \mathrm{m}$ (Figure 11 (B)). $1.50 \mathrm{~mA}$ current intensity results in $0.281 \mathrm{~V} / \mathrm{m}$ (Figure $11(\mathrm{C})$ ). And 2 $\mathrm{mA}$ current results into $0.375 \mathrm{~V} / \mathrm{m} \mathrm{EF}$ (Figure 11 (D)). Results indicates change in current intensity causes change in electric field distribution and strength in an individual.

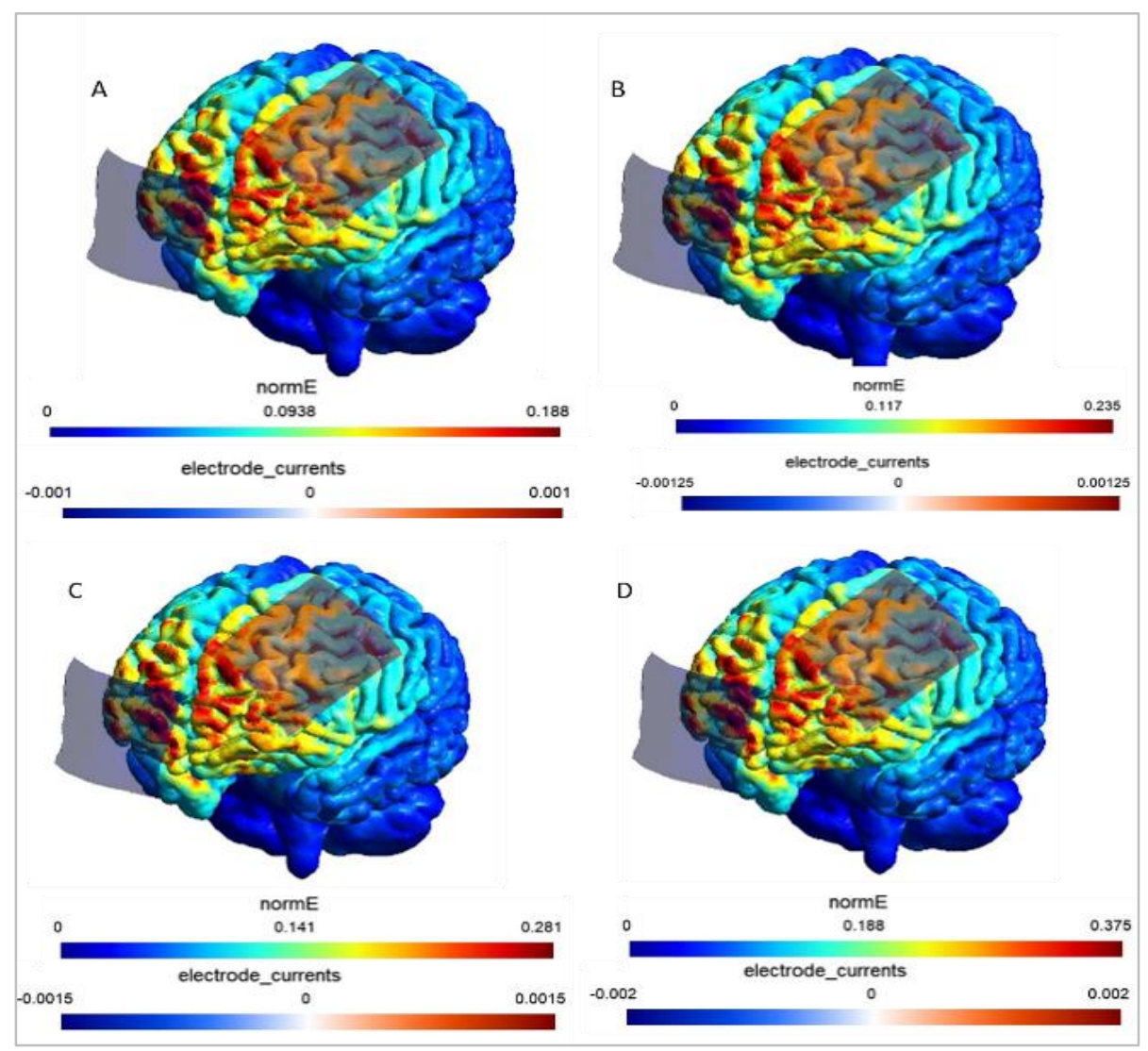

Figure 11 Intensities of Dose Current A) $1 \mathrm{~mA} \mathrm{~B}) 1.25 \mathrm{Ma} \mathrm{C}) 1.5 \mathrm{~mA} \&$ D) $2.0 \mathrm{~mA}$

\section{Discussion}

Computational modelling of the NIBS can help to shape treatment modality. Modelling of treatment with a computational approach helps to avoid unnecessary neuronal modulation at healthy sites. Electrode size and shape affect the focality of electric field strength. There are various parameters to be considered for modelling and change in each parameter can result in a varied electric field distribution. Size of an electrode can be selected according to patient head size also to increase or decrease focality. $5 \times 5 \mathrm{~cm} 2$ and $5 \times 7 \mathrm{~cm} 2$ are two standard sizes available for rectangular electrode. Elliptical shaped electrodes can also be modelled to know the change in electric field and focality. Electrode gel and connectors are other parameters for modelling and can be modelled with reference to standard tDCS devices available in the market. Input data required for simulation is MRI image which can be acquired with MRI machine for selected patients. Other alternative is to use MRI image data bank online which requires user authenticity for accessing the source data. Image segmentation done on the MRI images requires tools for separating different anatomical structures. Without image segmentation and processing accurate head models cannot be generated. There are tools available like ROAST, Bonsai modelling software, Spheres modelling software, SimNIBS and Comets. We have used SimNIBS as it has required software in one pipeline. Others may require additional software 
packages to process the tDCS simulation. Electrode placement requires knowledge about underlined structures according to the 10-20 electrode placement method. Majority of the tDCS research for the treatment of $\mathrm{AD}, \mathrm{MCI}$ and MDD have focused the LDLPFC as a target location for stimulation. Corresponding location was selected for LDLPFC is F3-anode and Fp2- cathode (Figure 4, Table 2 and Table 3). Alternative approach for identification of location is to define MNI co-ordinates for specific region. Detailed literature survey is required for MNI co-ordinates for accurate position of stimulating electrode. Generated electric field directly affected by the current reaching to the targeted brain tissues. There are multiple tissues present in a path of current and so the shunting of current. The shunted current and its induced electric field can be visualized in different brain tissues with one of the SimNIBS tool (Figure 9 and Figure 10). Maximum current shunted in a skull because it has greater density than rest of the brain tis sue. Electric field strength (normE) were calculated on gray matter (Region indices: 02, selected in SimNIBS). Peak electric fields were calculated and three different values obtained 0.184, 0.254 and 0.337 $\mathrm{V} / \mathrm{m}$ for 95,99 and 99.99 percentile of normE (For
AD). For MCI patient group 0.19, 0.258 and 0.341 $\mathrm{V} / \mathrm{m}$ obtained for 95,99 and 99.99 percentile of normE. Gray matter has two values for focality (Gray matter volume having peak electric fields greater than or equal to 50\% and 75\%) as shown in Table 5 and Table 6 for both $\mathrm{AD}$ and $\mathrm{MCI}$ patient group.

To alter the property of neuronal membrane with 0.2 $\mathrm{mV}$ of a threshold value, simulation results can be compared and validated. Keeping one stimulation parameter variable and the other being constant also helps to shape the treatment. We studied varying current intensity and its effects on individualized head models. We found that the strength of an electric field increases linearly with an increase in current intensity. Figure 12 shows the linear relationship between both the parameter. Evaluating graph we found that Increasing current intensity by $25 \%$ i.e. $1.25 \mathrm{~mA}$, we get $25 \%$ more electric field strength i.e. $0.235 \mathrm{~V} / \mathrm{m}$. And $50 \%$ increase in the current intensity results in $0.281 \mathrm{~V} / \mathrm{m}$, which is nearly $49.46 \%$ of increased electric field strength (normE). $100 \%$ increase in the current intensity results into $99.46 \%$ increase in the electric field strength i.e. $0.375 \mathrm{~V} / \mathrm{m}$.

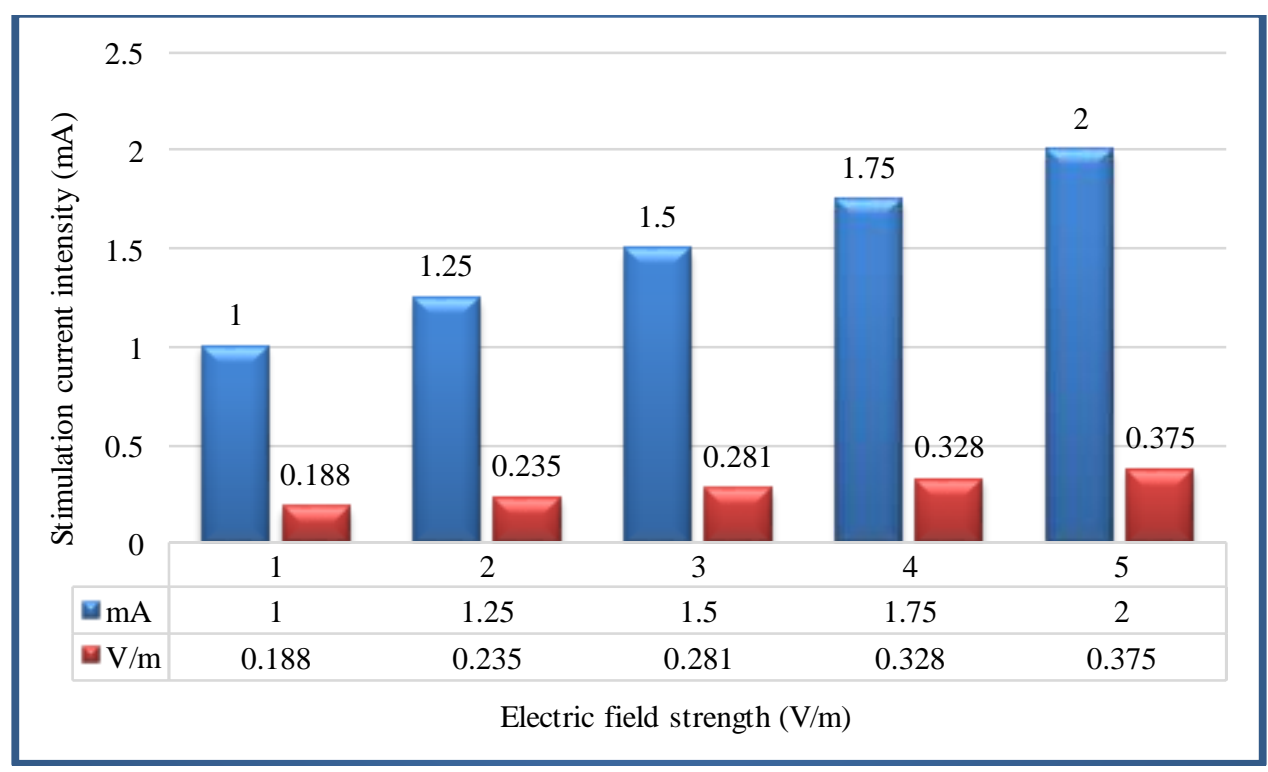

Figure 12 Graphical representation of current intensity vs. electric field strength

\section{Conclusion}

We have quantified Electric Field Distribution for AD and MCI patients upon tDCS stimulation. Appropriate selection of the parameters will decide the Electric Field Strength in a given subject. Current Intensity, Electrode's Shape and Size, Tissue Conductivities, Targeted Head Region according to 10-20 electrode placement method was selected for simulation. By estimating the electric field in both the subject for AD and MCI patients we got different normE or Electric Field Strength after tDCS stimulation. After simulation, we have high Electric Field Strength in MCI patients as they are in an early stage of dementia and related neurological disease. While in an $\mathrm{AD}$ 
patient as a disease is in a progressive stage and more damage happened to the brain tissue we assume that there is a significant loss of active neuronal population which results in poor electric field strength with same age group. Precise segmentation of DLPFC and other ROIs with separate mesh formations will help to understand more about EF distribution in a targeted area. This study is limited to a single patient fromeach disease group i.e. one from $\mathrm{AD}$ and the other from MCI. We have used two electrodes for simulation purposes. For the detailed study related to AD, the temporal lobe with multiple electrodes is required to understand the electric field strength. We have only studied varying stimulation parameters of current intensity in the proposed work, other stimulation parameters such as electrode shape, location, size, and orientation, the conductivity of tissues can also be studied with changes in detail. The future study includes patient-specific actual values of tissue conductivities with customized patient data from MRI and other imaging modalities. The study involves multiple patients for each patient group.

\section{Acknowledgment}

None.

\section{Conflicts of interest}

The authors have no conflicts of interest to declare.

\section{References}

[1] Alzheimer's Association. 2017 Alzheimer's disease facts and figures. Alzheimer's \& Dementia. 2017; 13(4):325-73

[2] Roberts R, Knopman DS. Classification and epidemiology of MCI. Clinics in Geriatric Medicine. 2013; 29(4):753-72.

[3] Andrade C, Arumugham SS, Thirthalli J. Adverse effects of electroconvulsive therapy. Psychiatric Clinics. 2016; 39(3):513-30.

[4] Ben-Menachem E. Vagus nerve stimulation, side effects, and long-term safety. Journal of Clinical Neurophy siology . 2001; 18(5):415-8.

[5] Prehn K, Flöel A. Potentials and limits to enhance cognitive functions in healthy and pathological aging by tDCS. Frontiers in Cellular Neuroscience. 2015; 9:114.

[6] Das S, Holland P, Frens MA, Donchin O. Impact of transcranial direct current stimulation (tDCS) on neuronal functions. Frontiers in Neuroscience. 2016; 10:1-7.

[7] Sharma G, Chowdhury SR. Enhancement in focality of non-invasive brain stimulation through high definition (HD) anodal transcranial direct current stimulation (TDCS) techniques. In conference on computational intelligence in bioinformatics and computational biology 2019 (pp. 1-5). IEEE.
[8] Marceglia S, Mrakic-Sposta S, Rosa M, Ferrucci R, Mameli F, Vergari M, et al. Transcranial direct current stimulation modulates cortical neuronal activity in Alzheimer's disease. Frontiers in Neuroscience. 2016; 10:134.

[9] Meinzer M, Lindenberg R, Phan MT, Ulm L, Volk C, Flöel A. Transcranial direct current stimulation in mild cognitive impairment: behavioral effects and neural mechanisms. Alzheimer's \& Dementia. 2015; 11(9):1032-40.

[10] Saturnino GB, Antunes A, Thielscher A. On the importance of electrode parameters for shaping electric field patterns generated by tDCS. Neuroimage. 2015; 120:25-35.

[11] Rodella C, Cespón J, Repetto C, Pellicciari MC. Customized application of TDCS in clinical rehabilitation of Alzheimer's Disease. Frontiers in Human Neuroscience. 2021; 15:1-4.

[12] Indahlastari A, Albizu A, O'Shea A, Forbes MA, Nissim NR, Kraft JN, et al. Modeling transcranial electrical stimulation in the aging brain. Brain Stimulation. 2020; 13(3):664-74.

[13] Vasavada MM, Martinez B, Wang J, Eslinger PJ, Gill DJ, Sun $X$, et al. Central olfactory dysfunction in Alzheimer's disease and mild cognitive impairment: a functional MRI study. Journal of Alzheimer's Disease. 2017; 59(1):359-68.

[14] Wang H, Wang X, Jin J, Zhang W, Li Y, Liu Z, Yin T. Simultaneous stimulation using RTMS and TDCS produces the most effective modulation of motor cortical excitability in healthy subjects: a pilot study. Neuroscience Letters. 2019; 694:46-50.

[15] Jog MV, Wang DJ, Narr KL. A review of transcranial direct current stimulation (tDCS) for the individualized treatment of depressive symptoms. Personalized Medicine in Psychiatry. 2019; 17:17-22.

[16] Hill AT, Rogasch NC, Fitzgerald PB, Hoy KE. Impact of concurrent task performance on transcranial direct current stimulation (tDCS)-Induced changes in cortical physiology and working memory. Cortex. 2019; 113:37-57.

[17] Laakso I, Mikkonen M, Koyama S, Hirata A, Tanaka S. Can electric fields explain inter-individual variability in transcranial direct current stimulation of the motor cortex? Scientific Reports. 2019; 9(1):1-10.

[18] Modolo J, Denoyer Y, Wendling F, Benquet P. Physiological effects of low-magnitude electric fields on brain activity: advances from in vitro, in vivo and in silico models. Current Opinion in Biomedical Engineering. 2018; 8:38-44.

[19] Hodgkin AL, Huxley AF. A quantitative description of membrane current and its application to conduction and excitation in nerve. The Journal of Phy siology. 1952; 117(4):500-44.

[20] Brunel N, Van Rossum MC. Lapicque's 1907 paper: from frogs to integrate-and-fire. Biological Cybernetics. 2007; 97(5):337-9.

[21] Lee S, Lee C, Park J, Im CH. Individually customized transcranial temporal interference stimulation for focused modulation of deep brain structures: a 
simulation study with different head models. Scientific Reports. 2020; 10(1):1-11.

[22] Saturnino GB, Puonti O, Nielsen JD, Antonenko D, Madsen KH, Thielscher A. SimNIBS 2.1: a comprehensive pipeline for individualized electric field modelling for transcranial brain stimulation. Brain and Human Body Modeling. 2019:3-25.

[23] Windhoff M, Opitz A, Thielscher A. Electric field calculations in brain stimulation based on finite elements: an optimized processing pipeline for the generation and usage of accurate individual head models. Hoboken: Wiley Subscription Services. 2013:923-35.

[24] Jurcak V, Tsuzuki D, Dan I. 10/20, 10/10, and 10/5 systems revisited: their validity as relative headsurface-based positioning systems. Neuroimage. 2007; 34(4):1600-11.

[25] Neulinger K, Oram J, Tinson H, O'Gorman J, Shum DH. Prospective memory and frontal lobe function. Aging, Neuropsychology, and Cognition. 2016; 23(2):171-83.

[26] Berlucchi G, Vallar G. The history of the neurophysiology and neurology of the parietal lobe. Handbook of Clinical Neurology. 2018; 151:3-30.

[27] Clark RE. Current topics regarding the function of the medial temporal lobe memory system. Behavioral Neurosciences.2018:1-30.

[28] Javed K, Reddy V, Lui F. Neuroanatomy, cerebral cortex. StatPearls Publishing.2019.

[29] Mollet GA. Fundamentals of human neurop sy chology. Journal of Undergraduate Neuroscience Education. 2008; 6(2): R3-R4.

[30] Lezak MD, Howieson DB, Loring DW, Fischer JS. Neuropsychological assessment. Oxford University Press, USA; 2004.

[31] Mendoza J, Foundas A. Clinical neuroanatomy: a neurobehavioral approach. Springer Science \& Business Media; 2007.

[32] Von Bartheld CS, Bahney J, Herculano-Houzel S. The search for true numbers of neurons and glial cells in the human brain: a review of 150 years of cell counting. Journal of Comparative Neurology. 2016; 524(18):3865-95.

[33] Seibt O, Brunoni AR, Huang Y, Bikson M. The pursuit of DLPFC: non-neuronavigated methods to target the left dorsolateral pre-frontal cortex with symmetric bicephalic transcranial direct current stimulation (tDCS). Brain Stimulation. 2015; 8(3):590-602.

[34] Bunai T, Hirosawa T, Kikuchi M, Fukai M, Yokokura $\mathrm{M}$, Ito $\mathrm{S}$, et al. tDCS-induced modulation of GABA concentration and dopamine release in the human brain: a combination study of magnetic resonance spectroscopy and positron emission tomography. Brain Stimulation. 2021; 14(1):154-60.

[35] André S, Heinrich S, Kayser F, Menzler K, Kesselring $\mathrm{J}$, Khader PH, et al. At-home tDCS of the left dorsolateral prefrontal cortex improves visual shortterm memory in mild vascular dementia. Journal of the Neurological Sciences. 2016; 369:185-90.
[36] Holczer A, Németh VL, Vékony T, Vécsei L, Klivényi $\mathrm{P}$, Must A. Non-invasive Brain Stimulation in Alzheimer's disease and mild cognitive impairment-a state-of-the-art review on methodological characteristics and stimulation parameters. Frontiers in Human Neuroscience. 2020; 14:179.

[37] Nitsche MA, Doemkes S, Karakose T, Antal A, Liebetanz D, Lang N, et al. Shaping the effects of transcranial direct current stimulation of the human motor cortex. Journal of Neurophysiology. 2007; 97(4):3109-17.

[38] Bhattacharjee S, Kashyap R, Rapp B, Oishi K, Desmond JE, Chen SA. Simulation analyses of tDCS montages for the investigation of dorsal and ventral pathways. Scientific Reports. 2019; 9(1):1-17.

[39] https://simnibs.github.io/simnibs/build/html/index.htm 1. Accessed 26 May 2021.

[40] J. Peters, G. Stinstra, M. Hendriks M. Estimation of the electrical conductivity of human tissue. Electromagnetics. 2001; 21(7-8):545-57.

[41] Opitz A, Paulus W, Will S, Antunes A, Thielscher A. Determinants of the electric field during transcranial direct current stimulation. Neuroimage. 2015; 109:14050.

[42] Wagner TA, Zahn M, Grodzinsky AJ, Pascual-Leone A. Three-dimensional head model simulation of transcranial magnetic stimulation. IEEE Transactions on Biomedical Engineering. 2004; 51(9):1586-98.

[43] Laakso I, Tanaka S, Mikkonen M, Koy ama S, Sadato $\mathrm{N}$, Hirata A. Electric fields of motor and frontal tDCS in a standard brain space: a computer simulation study. Neuroimage. 2016; 137:140-51.

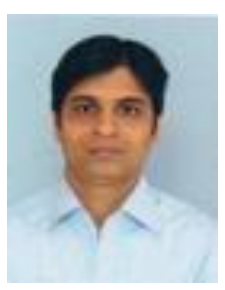

Utkarsh Pancholi is currently an Assistant Professor in the Biomedical Engineering Department, L.D College of Engineering affiliated with Gujarat Technological University. He has done M.Tech from IIT, BHU. Varanasi, India. His area of interest is Computational Neuroscience, Neuromodulation, and

Rehabilitation.

Email: utkarshpancholi6387@gmail.com

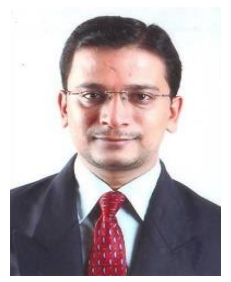

Vijay Dave is currently an Assistant Professor in Biomedical Engineering Department, GEC, Gandhinagar, Gujarat affiliated with Gujarat Technological University. He has done M.Tech and Ph.D. from IIT, Mumbai, Maharastra, India. His area of interest is Computational Neuroscience, Biopotential Instrumentation and Rehabilitationn Technology.

Email: vijaydave12@gmail.com 
Utkarsh Pancholi and Vijay Dave

\begin{tabular}{|c|c|c|}
\hline \multicolumn{3}{|c|}{ Appendix I } \\
\hline S.No. & - Abbreviation & Description \\
\hline 1 & 3D & Three Dimensional \\
\hline 2 & $\mathrm{AD}$ & Alzheimer's Disease \\
\hline 3 & ADNI & $\begin{array}{l}\text { Alzheimer's Disease Neuroimaging } \\
\text { Initiative }\end{array}$ \\
\hline 4 & $\mathrm{Ca}+$ & Calcium positive ion \\
\hline 5 & CAT & Computational Anatomy Toolbox \\
\hline 6 & $\mathrm{~cm}$ & Centimeter \\
\hline 7 & CSF & Cerebrospinal Fluid \\
\hline 8 & ECT & Electro-convulsive therapy \\
\hline 9 & EEG & Electroencephalography \\
\hline 10 & $\mathrm{EF}$ & Electric Field \\
\hline$\pi 1$ & fMRI & $\begin{array}{l}\text { Functional Magnetic } \text { Resonance } \\
\text { Imaging }\end{array}$ \\
\hline 12 & GABA & Gamma-Aminobutyric Acid \\
\hline 13 & GM & Gray Matter \\
\hline 14 & gmsh & $\begin{array}{l}\text { Open source 3D finite element mesh } \\
\text { generator }\end{array}$ \\
\hline 15 & GR & Gradient (Pulse Sequence) \\
\hline 16 & LDLPFC & Left Dorsolateral Prefrontal Cortex \\
\hline 17 & LTD & Long Term Depression \\
\hline 18 & LTP & Long Term Potentiation \\
\hline 19 & $\mathrm{~mA}$ & Milliampere \\
\hline 20 & MCI & Mild Cognitive Impairment \\
\hline 21 & MDD & Major Depressive Disorder \\
\hline 22 & $\mathrm{~mm}$ & Millimeter \\
\hline 23 & MNI & Montreal Neurological Institute \\
\hline 24 & MRI & Magnetic Resonance Imaging \\
\hline 25 & $\mathrm{~ms}$ & Millisecond \\
\hline 26 & NIBS & Non-ınvasive Brain Stimulation \\
\hline 27 & NifT I & $\begin{array}{l}\text { Neuroimaging InformaticsTechnology } \\
\text { Initiative }\end{array}$ \\
\hline 28 & ROAST & $\begin{array}{l}\text { Realistic, Volumetric Approach to } \\
\text { Simulate Transcranial } \text { Electric } \\
\text { Stimulation }\end{array}$ \\
\hline 29 & rTMS & $\begin{array}{l}\text { Repetitive Transcranial Magnetic } \\
\text { Stimulation }\end{array}$ \\
\hline 30 & $\mathrm{~S} / \mathrm{m}$ & Siemens per Meter \\
\hline 31 & SimNIBS & $\begin{array}{l}\text { Simulation of Non-invasive Brain } \\
\text { Stimulation }\end{array}$ \\
\hline 32 & SPM & Statistical Parametric Mapping \\
\hline 33 & tDCS & $\begin{array}{lll}\text { Transcranial } & \text { Direct } & \text { Current } \\
\text { Stimulation } & & \\
\end{array}$ \\
\hline 34 & TE & Echo Time \\
\hline 35 & TI & Inversion Time \\
\hline 36 & TMS & Transcranial Magnetic Stimulation \\
\hline 37 & $\mathrm{TR}$ & Repetition Time \\
\hline 38 & $\mathrm{~V} / \mathrm{m}$ & Volts per Meter \\
\hline 39 & $\mathrm{WM}$ & White Matter \\
\hline
\end{tabular}

